\title{
Analysis of Wigner's Set Theoretical Proof for Bell-Type Inequalities
}

\author{
Karl Hess ${ }^{1}$, Hans De Raedt ${ }^{2}$, Kristel Michielsen ${ }^{3 *}$ \\ ${ }^{1}$ Center for Advanced Study, University of Illinois, Urbana, IL, USA \\ ${ }^{2}$ Zernike Institute for Advanced Materials, University of Groningen, Groningen, The Netherlands \\ ${ }^{3}$ Institute for Advanced Simulation, Jülich Supercomputing Centre, Forschungszentrum Jülich, Germany RWTH Aachen University, \\ Aachen, Germany \\ Email: *k.michielsen@fz-juelich.de
}

How to cite this paper: Hess, K., De Raedt, H. and Michielsen, K. (2017) Analysis of Wigner's Set Theoretical Proof for BellType Inequalities. Journal of Modern Physics, 8, 57-67.

http://dx.doi.org/10.4236/jmp.2017.81005

Received: December 13, 2016

Accepted: January 7, 2017

Published: January 10, 2017

Copyright (C) 2017 by authors and Scientific Research Publishing Inc. This work is licensed under the Creative Commons Attribution International License (CC BY 4.0). http://creativecommons.org/licenses/by/4.0/

\begin{abstract}
We present a detailed analysis of the set theoretical proof of Wigner for Bell type inequalities with the following result. Wigner introduced a crucial assumption that is not related to Einstein's local realism, but instead, without justification, to the existence of certain joint probability measures for possible and actual measurement outcomes of Einstein-Podolsky-Rosen (EPR) experiments. His conclusions about Einstein's local realism are, therefore, not applicable to EPR experiments and the contradiction of the experimental outcomes to Wigner's results has no bearing on the validity of Einstein's local realism.
\end{abstract}

\section{Keywords}

Bell Inequality, Local Realism, Nonlocality

\section{Introduction}

Einstein challenged the Copenhagen interpretation of quantum mechanics by proposing with Podolsky and Rosen [1] Gedanken-Experiments, briefly called EPR experiments. These experiments were to demonstrate that quantum mechanics is incomplete, having missed in its description of physical reality some elements of that reality.

About 30 years after the EPR paper, Bell [2] derived an inequality for functions of elements of physical reality, known since as Bell's inequality, that in his opinion had to be obeyed by all of classical physics, meaning in essence by the framework of Einstein's relativity. Wigner transformed Bell's derivation into a set theoretical approach [3].

The work of Bell and his followers had very important consequences for the views on the foundations of physics. A majority of physicists believe that either (i) any physical theory using counterfactually definite functions and obeying Einstein's local realism (see II) must obey Bell-type inequalities and/or (ii) Wigner's derivation of Bell-type 
inequalities is only based on set theory (not involving counterfactual reasoning) and Einstein's local realism. There is a considerable body of serious mathematical-physical work that has raised objections against both (i) and (ii) (see particularly [4] and references therein as well as [5] and [6]). We have shown in [7] that (i) is false and it is the purpose of this paper to show that (ii) is also false.

The belief in (ii) arose mainly from the work of Wigner [3] and a popularized version of Wigner's work by d'Espagnat [8]. (d'Espagnat, however, also uses counterfactual reasoning that raises additional questions [7].) The fact that many EPR-types of experiments have been published that violate Bell's and Wigner's inequalities, is therefore presented as proof against the validity of Einstein's local realism, because it appears inconceivable that set theory is incorrect.

There exists, however, the following problem with this latter assessment. Set theory represents a mathematical framework based on axioms that may be regarded as definitions. As such, set theory is not related to any measurements and experiments and, therefore, also not to EPR experiments. It is thus necessary to find a connection between the sets of elements of physical reality that the experiments provide and the sets of mathematical abstractions that Wigner uses in his proof. We analyze this connection in great detail and show that Wigner's sets of mathematical abstractions contain a topological-combinatorial ordering that is incompatible with the actual ordering of measurement-events in space and time (or space-time). We suggest that it is this incompatibility that leads to the contradictions of Wigner's work with actual EPR measurements and not any failure of Einstein's local realism. An analogous situation was discussed by Poincaré and Einstein, who resolved the experimental contradictions to Euclidean geometry by pointing to the connection of Euclidean geometry with the physical reality, a connection achieved by the introduction of the concept of rigid bodies [9].

As we will show, there exists an analogy to the Einstein-Poincaré discussions, because Wigner connected his mathematical sets to the actual measurements in a way that is inconsistent with the space-time physics of the actual EPR experiments. Key to our reasoning is the fact that the set of data of the actual measurements is necessarily involving space- and time- (or space-time-) coordinates to designate the correlated pairs. These sets of space and time coordinates are inconsistent with Wigner's sets and subsets that are ordered according to location in three-dimensional space and equipment settings in that space, but do not involve measurement times.

We show in detail that Wigner's derivation of Bell type inequalities (that restrict the possible correlations exhibited by EPR measurements) does actually not use Einstein's local realism at all. Instead Wigner assumes in a hidden and unjustified way certain topological combinatorial properties of his mathematical sets that are inconsistent with the macroscopic properties of the measurement equipment in space and time as determined by clocks, protractors and meter-measures. The contradictions between Wigner's (Bell-type) inequalities and well known experiments (see II B) must, therefore be blamed on Wigner's topological-combinatorial assumptions and not on Einstein's local realism.

To prove this finding, we first analyze the elements of physical reality used in EPR 
experiments and form the sets of physical elements that must be dealt with in Wigner's mathematical proof (see Section 2). We then review the essential portion of Wigner's mathematical proof and point to his unwarranted assumptions (see Section 3). Finally, in Section 4 we deduce from these latter assumptions the topological-combinatorial problems of Wigner's work and conclude that the well known line "death by experiment" applies to Wigner's theory not to Einstein's. Our conclusions are summarized in Section 5 .

\section{Einstein's Realism, EPRB Experiments and Their Resulting Data}

We consider in the following only a variation of EPR experiments as suggested by Bohm and, therefore called EPRB experiments [10] [11]. This type of experiment involves the measurement of the two single entities of correlated pairs of particles. Each single entity is measured at a different set of space-coordinates. These space-coordinates must, from the viewpoint of physics, describe the entire local measurement equipment for both measurement locations. For brevity and because important measurements have actually been performed there, we will denote the two measurement locations and corresponding space coordinates just by "Tenerife" and "La Palma". The space-like separation of the measurements is a necessity to invoke Einstein's separation principle, which is an important factor of the EPR argument and focus of the EPR discussion. Einstein's separation principle excludes all influences between the two measurement stations that are faster than the speed of light in vacuum. This principle guarantees a certain independence of the two separated measurement stations and represents the core of what is commonly called Einstein's local realism. A broader discussion and detailed definition of Einstein's local realism has been given by Fine [12].

It is, therefore, of utmost importance for the discussions of Bell and Wigner to exclude in the actual experiments influences from the other location that propagate with speed slower or equal to that light. How can one make sure that there exist no such other influences and in addition that one indeed measures correlated pairs? This goal has been achieved in [10], [11] and other experiments. These measurements relate to the Clauser-Horn-Shimony-Holt (CHSH) inequality. We note here, however, that all of our reasoning applies, as will become evident below, also to this type of inequality. We just discuss the Bell inequality in our analysis, because Wigner's proof referred only to this type of inequality.

\subsection{Switching Instruments and Pairing Particles}

Key to Bell's and Wigner's reasoning is the rapid switching of instruments on both Tenerife and La Palma, just before a measurement of the spin of an incoming particle is performed. In this way one ensures that the instrument settings on the other island cannot influence a given measurement with the speed of light or lower speeds. What Bell and Wigner wished to find out was whether Einstein's local realism is correct or incorrect. The importance of this fast switching is emphasized over and over by Bell [2] in his discussions, but no trace of it can be found in either Bell's or Wigner's formalism 
and algebra of their actual proofs. The reason for this obvious lack of correspondence between theory and experiment is the assumption of Bell and all his followers that the possible outcomes of pair-measurements are just a given for their mathematical abstractions (functions, sets) and the fact how the pairing experimentally occurred is not included in the theory.

However, it is precisely this pairing and timing problem, where Einstein embedded the necessity of using a space-time system, his space-time system, as the basis for his Gedanken-Experiments and the theoretical approach with Podolsky and Rosen, [1], [13]. There also exists no other known way to pair and correlate space like separated measurement outcomes than by space and time measurements. ${ }^{1}$

The timing of the measurements is, thus, a most important element of the physical reality, of the data, and we denote the clock-time of the $n$th measurement in Tenerife by $t_{n}$ and that in La Palma by $t_{n}^{\prime}$. Note that subsequent measurement pairs are necessarily time-like separated because the switching of the macroscopic settings cannot be instantaneous. To identify actual correlated pairs, some rule needs to be employed that selects the pairs. An example of such a rule would be that the time difference of the measurements at the two different locations (islands) must be smaller than a certain value $W$. We emphasize that through any such rule an instantaneous logical and physical connection is established between the measurements at the two different locations that has nothing to do with instantaneous influences, much less with information transfer. Without such a connection, no correlations between the spacelike separated measurements can be established. Only with such a connection can intricate correlations due to physical law be revealed that now may involve also the many-body dynamics of the measurement equipment of both locations. It also must be emphasized that the analysis of the raw data of three different EPRB experiments [11] [14] [15] [16] do show a significant $W$-dependence of the amount of violations of Bell-type inequalities (actually the CHSH inequality), for values of $W$ that are much smaller than the average time between the detection of photons [17] [18].

From what follows in this paper, it appears imperative to perform measurements that involve dependencies on the timing and on the instrument settings in relation to that timing.

Wigner ignored the measurement times and corresponding connections when considering possible and actual outcomes in his theory and we will see that this negligence has serious consequences for his set theoretic calculations of correlations.

\subsection{Elements of Physical Reality: Notation and Notebook-Entries}

Actual EPRB measurements (e.g. [10] [11]) monitor, in addition to the clock-times of

${ }^{1}$ We do not wish to use quantum-physics arguments in our reasoning. However, it must be said that the nature of the experiments and the possibility of quantum fluctuations excludes any other method of pairing and leaves only space and time measurements. Suppose, for example, that we just count on each island the number of measurements and then assume that those with equal number (deduced from the counting) will be selected as correlated pairs (post measurement, of course). Then a single counting error (caused for example by a quantum fluctuation in the measurement equipment) will completely mess up the ordering and will lead to pairing of the wrong entities. One must, therefore, use synchronized clocks in the space-like separated measurement stations, as well as knowledge about the space-like separation in order to identify which detector clicks represent, with high likelihood, measurements of a correlated pair. 
the detector events, two equipment orientations that determine the spin (polarization) of incoming particles. These registrations, for photons often obtained with polarizers and avalanche photodiodes, have been denoted by a variety of symbols such as horizontal and vertical etc. and Wigner uses + and - for any particles that he considers. We denote the measurement result for the $n$th measurement in Tenerife by $s_{n}$ and that in La Palma by $s_{n}^{\prime}$ each being either - or + .

As mentioned, the atoms and molecules constituting the measurement equipment have many body interactions with the incoming particles, from both the view of Einstein's physics and also from the most modern view of quantum physics. Nevertheless, both Bell, Wigner and all their followers describe this equipment, as the Copenhagen school of quantum mechanics does, just by a three-dimensional unit vector of space, indicating the direction of a polarizer or of a Stern-Gerlach magnet. We denote this unit vector for the nth experiment by $j_{n}$ in Tenerife and $j_{n}^{\prime}$ in La Palma, respectively. Wigner assumed that this unit vector may assume precisely the same three directions $a, b, c$ in both Tenerife and La Palma and that these directions are randomly chosen on each island in the actual experiments. Using these assumptions and notational conventions we arrive at EPRB notebook-entry pairs of the kind:

$$
\left[s_{n}, j_{n}, t_{n} ; s_{n}^{\prime}, j_{n}^{\prime}, t_{n}^{\prime}\right]
$$

where, as mentioned, both $s_{n}$ and $s_{n}^{\prime}$ may (exclusively) assume the value of either + or - and both $j_{n}$ and $j_{n}^{\prime}$ are randomly chosen from the vectors $a, b, c$. The parenthesis [.] indicates the measurement of a correlated pair and the symbol ";" denotes the separation of entries for Tenerife and La Palma. The number 9 of the time-selected pairs thus could be represented by the following actual notebook entry $\left[+, a, t_{9} ;-, b, t_{9}^{\prime}\right]$. As far as EPRB experiments are concerned such entries are collections of elements of physical reality (in the sense of Mach) and represent the only physical reality that we need to consider to discuss Wigner's proof.

As mentioned, both Bell and Wigner use Einstein locality as a tool of reasoning: For carefully designed EPRB experiments they reason, that the measurement outcome $s_{n}$ does not depend on what vector $j_{n}^{\prime}$ is chosen in La Palma and $s_{n}^{\prime}$ does not depend on $j_{n}$ in Tenerife.

Wigner discussed in his paper three pairs of measurements with the given settings $[a, b],[a, c]$ and $[b, c]$, exactly those that Bell had chosen for his inequality. Such a selection gives us the following six-tuple that we call henceforth Bell's six-tuple:

$$
\left[s_{k}, a, t_{k} ; s_{k}^{\prime}, b, t_{k}^{\prime}\right]\left[s_{l}, a, t_{l} ; s_{l}^{\prime}, c, t_{l}^{\prime}\right]\left[s_{m}, b, t_{m} ; s_{m}^{\prime}, c, t_{m}^{\prime}\right] .
$$

Here $k=1, \cdots, M, l=M+1, \cdots, 2 M$, and $m=2 M+1, \cdots, 3 M$ where $M$ is a large number determined by the total number of measurements. The brackets [.] indicates again the measurement of a correlated pair. If we assume that these pairs are derived from the notebook entries (1), then Einstein's local realism does not put any constraint on the possible values of the pairs $s_{k}, s_{k}^{\prime}, s_{l}, s_{l}^{\prime}$ and $s_{m}, s_{m}^{\prime}$. For given measurement times, they may assume any of the possible $2^{6}$ combinations of values of + and - , because from both a physical and mathematical point of view there are at this point no restrictions to the possible outcomes except that they are two-valued. 
For each pair, we are interested with Wigner in only two different possibilities when measuring on the individual particles: either equal outcomes or unequal outcomes. Therefore, for a string of $M$ measurements of pairs, we have $2^{M}$ different possible outcomes. If we have three such strings of pairs we have $2^{3 M}$ possible different correlations of the pair outcomes. Wigner reduces the number of correlations that he considers essential significantly further, by counting only those that yield a different fraction of the final count of equal relative to unequal outcomes. Simple counting leads then to the conclusion that for each string of $M$ measurements of pairs we have $M+1$ different possible outcomes that lead to a different fraction of equal and unequal outcomes (see Table 1 for an illustration of the counting procedure). Therefore we obtain for the six-tuple (2) $(M+1)^{3}$, possible outcomes that Wigner counts as different. $^{2}$

As we have mentioned, Einstein's local realism has not been used up to now. We show below that it also is not used in Wigner's further considerations that lead to his variation of Bell's inequality. Wigner's theory does thus derive, without reference to Einstein locality, how many different correlations of equal and different outcomes may occur for the $3 M$ pairs of measurements. Nevertheless, Wigner's number of possible different correlations is significantly reduced below the above mentioned value of $(M+1)^{3}$ and is only proportional to $M^{2}$.

We ask, therefore, the question: What caused the reduction of the possible number of correlations in Wigner's proof? Our answer is that it was not any assumption regarding Einstein's local realism. Instead, the reduction of the number of possible correlations arises from Wigner's unwarranted mathematical assumption that is related to set

Table 1. Fictive example of all possible notebook entries of an experiment with $M=3$ measurements per setting, adopting Wigner's method of counting. The "e" (" $u$ ") in columns $C_{1}, C_{2}$, and $C_{3}$ indicate that a pair yields outcomes that are equal (unequal) for measurements of the first, second and third pair of settings, respectively. The last column labeled "e/u" gives the fraction of equal and unequal correlations $C_{1}, C_{2}$, and $C_{3}$. The maximum number of different items in the last column is given by $M+1=4$ in this particular example.

\begin{tabular}{cccc}
\hline$C_{1}$ & $C_{2}$ & $C_{3}$ & $\mathrm{e} / \mathrm{u}$ \\
\hline $\mathrm{e}$ & $\mathrm{e}$ & $\mathrm{e}$ & $3 / 0$ \\
$\mathrm{u}$ & $\mathrm{u}$ & $\mathrm{e}$ & $2 / 1$ \\
$\mathrm{u}$ & $\mathrm{u}$ & $\mathrm{u}$ & $0 / 3$ \\
$\mathrm{e}$ & $\mathrm{u}$ & $\mathrm{u}$ & $1 / 2$ \\
$\mathrm{e}$ & $\mathrm{e}$ & $\mathrm{u}$ & $2 / 1$ \\
$\mathrm{u}$ & $\mathrm{e}$ & $\mathrm{u}$ & $1 / 2$ \\
$\mathrm{u}$ & $\mathrm{u}$ & $\mathrm{e}$ & $1 / 2$ \\
$\mathrm{u}$ & $\mathrm{e}$ & $\mathrm{e}$ & $2 / 1$ \\
\hline
\end{tabular}

\footnotetext{
${ }^{2}$ The mathematical proof for this fact is easily performed by writing out the possible different outcomes in matrix-form (first column all equal, last all different) and using the method of complete induction assuming correctness for $M$ and proving the result for $M+1$. We have previously given a very transparent proof by using the numbers +1 and -1 for the possible equal and different pair-outcomes respectively [8].
} 
theoretic probability theory. The reduction of possible correlations is, therefore, artificial and does not apply to EPR experiments. As mentioned, the celebrated line "death by experiment" is correct but it applies to Wigner's theory and not, as reported in so many sensationalist articles, to Einstein's.

\section{Wigner's Additional Assumptions}

Wigner did not consider any dependence of the measurement outcomes on the measurement times and just assumed that the possible or actual outcomes $s_{n}$ and $s_{n}^{\prime}$ involve automatically, so to speak per fiat, a correlated pair. The measurement locations and times have, however, topological-combinatorial consequences for the sets of elements of physical reality that are represented by Wigner's sets of mathematical abstractions. It is just these latter consequences that we discuss here. ${ }^{3}$

Wigner defines (p. 1007, left column last paragraph of [3]) "domains of the space of the hidden variables" and claims (in agreement with our thinking about possible outcomes outlined above) that "we have only $2^{6}$ essentially different domains". These can be characterized by symbols:

$$
\left(\sigma_{1}, \sigma_{2}, \sigma_{3} ; \tau_{1}, \tau_{2}, \tau_{3}\right),
$$

all $\sigma$ and $\tau$ assuming two possible values: + or - , and the $\sigma$ referring to the first, the $\tau$ to the second, particle. Wigner's detailed explanations also point out that the domains characterized by $\left(\sigma_{1}, \sigma_{2}, \sigma_{3} ; \tau_{1}, \tau_{2}, \tau_{3}\right)$ correspond precisely to the respective equipment-settings $(a, b, c ; a, b, c)$ which leads to the 9 possible pairings (see Equaton (6) below) from which the possible pairs of Bell's six-tuple (2) and possible outcomes $\left(s_{k}, s_{k}^{\prime}, s_{l}, s_{l}^{\prime}\right.$ and $s_{m}, s_{m}^{\prime}$ in our notation) can be selected.

In the next paragraph Wigner states his most crucial assumption by a definition: "Let $\left(\sigma_{1}, \sigma_{2}, \sigma_{3} ; \tau_{1}, \tau_{2}, \tau_{3}\right)$ denote henceforth the probability that the hidden parameters assume, for the singlet state of the two spins, a value lying in the domain which was denoted by this symbol". As innocuous as this definition looks, it contains a very big assumption, the assumption of the existence of a joint probability for the six-tuples (3) of possible outcomes of the measurements, while the actual measurements are only performed in pairs.

Wigner's assumption about the joint probability of the domains of variables (parameters) that determine the possible outcomes implies the existence of consistent joint probabilities for the possible and actual outcomes of measurements such as $(+,-,-;-,+,-)$. Note, however, that these possible outcomes are now assumed to be listed in six-tuples with the setting-sequence of $a, b, c$ on each island, in spite of the fact that actually only pairs are measured. From a set theoretic viewpoint we must ask ourselves: Which sets is Wigner discussing? Without doubt these must be sets of measurements of time-correlated pairs.

As an aside, Bernard d'Espagnat [8] presented a counterfactual interpretation of Wigner's work by introducing multiple hypothetical values that every single particle "possesses" and that are assumed to exist in addition to the possible measurement

${ }^{3}$ The Bell's reasoning starts to deviate here from Wigner because of his introduction of counterfactually definite functions. We have dealt with this approach in connection with a many body dynamics of particle-equipment interactions in [7]. 
outcomes for particle pairs. We do not make these additional unwarranted assumptions. Our reasoning below does, however, also apply to d'Espagnat's work, with the addition of our findings about counterfactual approaches in [7].

Wigner himself intended, as did Bell originally, to use exclusively elements of physical reality in the sense of Mach and we discuss his work from this point of view only.

\section{Problems with Wigner's Set-Theoretical Assumptions}

As an illustration of how far reaching Wigner's assumptions are, consider Bell's six-tuple (2) which contains three correlated pairs and add to each of the pairs an arbitrary (artificial) third notebook entry for the setting that is not already contained in the pair-outcomes: setting $c$ for the first pair, $b$ for the second and $a$ for the third. As an example we obtain then for the first pair-column a triple-column: ${ }^{4}$

$$
\left[s_{k}, a, t_{k} ; s_{k}^{\prime}, b, t_{k}^{\prime}\right] \rightarrow\left(s_{k}, a, t_{k} ; s_{k}^{\prime}, b, t_{k}^{\prime} / s_{?}, c, t_{?}\right) .
$$

The ? as subscripts indicate the arbitrariness of choice of the additions. We have separated these additions by "/" instead of "; ", because the latter indicates the separation of the measurements on two islands and it is not determined from which island the additions originate. The three entries are also not all correlated in time, because $t_{\text {? }}$ cannot equal any of the other measurement times of the triple. Different settings must correspond to different times in Einstein's physics. We did not use the symbol [.] on the right side of (4) but used instead (.), because the pairing by measurement times cannot be guaranteed for all of them. Together with the other two pairs of the Bell six-tuple, we obtain thus three triples each with settings $a, b, c$. All the followers of Bell and Wigner, not just d'Espagnat but also Leggett-Garg [19] and others, assume explicitly or implicitly that the joint triple probabilities exist and are common to all three triples.

However, it is a well known fact of probability theory, particularly the set theoretic probability theory of Kolmogorov, that the existence of joint triple (and higher order) probabilities is not guaranteed [4], [13]. In the above example, each column of triples may, for example, have different joint triple probabilities and a joint triple probability that is common to all three columns of triples may not exist. The proof of the validity of Bell type inequalities requires an existence proof of joint triple (quadruple etc.) probabilities from the underlying physics. Wigner just assumed these probabilities to exist and, therefore, assumed what he had to prove.

This is an enormously important point that has been overlooked also by Bell and all his followers [2]. If one claims that Wigner's approach is a set theoretic approach, we need to use a set theoretic definition of probability measures. Such a definition indeed exists and is, as mentioned, given by the probability framework of Kolmogorov [20]. That framework teaches us that, for a countable number of considered possible outcomes such as $\left(\sigma_{1}, \sigma_{2}, \sigma_{3} ; \tau_{1}, \tau_{2}, \tau_{3}\right)$, one can define consistent joint probabilities if and only if the actual measurements can also be listed or taken in form of such six-tuples so that we can establish a one to one correlation of any of Wigner's $\sigma, \tau$ with the ${ }^{4}$ This is precisely what d'Espagnat did in his variation on the theme of Wigner (see p162 of his essay in Scientific American [8]). 
(possible) measurement outcomes $s_{n}$ and $s_{n}^{\prime}$. Wigner has thus made a mathematical assumption of serious consequences without having any physical or mathematical reason.

Several different cases need to be considered to present the full proof that Wigner's approach must not be applied to EPRB experiments. We just present one example that is typical and list the following six-tuple using Wigner's subsets related to settings $a, b, c$ for both Tenerife and La Palma with a possible choice of measurement times included:

$$
\left(s_{h}, a, t_{h} / s_{i}, b, t_{i} / s_{j}, c, t_{j} ; s_{i}^{\prime}, a, t_{i}^{\prime} / s_{j}^{\prime}, b, t_{j}^{\prime} / s_{h}^{\prime}, c, t_{h}^{\prime}\right)
$$

The symbol "/" separates the three Wigner subsets on one given island. The indexes $h, i, j$, represent natural numbers $1,2,3 \cdots$ that are now different from those used previously $(k, l, m)$ in the six-tuple (2). The reason for using a different notation is that six-tuples (2) and (5) cannot be transformed into each other without violating the space and time correlations of the actual pairing arising from the original measurements. The particular chosen pairing of six-tuple (5) is determined by the measurement times and thus is given by $[a ; c],[b ; a]$ and $[c ; b]$, while the pairing of six-tuple (2) is $[a ; b],[a ; c]$ and $[b ; c]$ and the pairing by measurement times must be different whenever the topology (location and setting) of the measurements of the pairs is different.

Wigner never included considerations of both measurement times and topology and used six-tuple (5) to obtain the possible outcomes for all the following 9 possible pairings:

$$
(a ; a),(a ; b),[a ; c],[b ; a],(b ; b),(b ; c),(c ; a),[c ; b],(c ; c) .
$$

We use again [.] only for the guaranteed correlated pairs (through measurement times). For all other pairs we use $(\cdot)$. For the pairings of Bell's six-tuple (2) and using (3) Wigner thus obtains:

$$
\left(\sigma_{1}, a ; \tau_{2}, b\right)\left[\sigma_{1}, a ; \tau_{3}, c\right]\left(\sigma_{2}, b ; \tau_{3}, c\right) .
$$

The $\sigma$ and $\tau$ denote here a certain given outcome of either + or - . For example, we may have $\sigma_{1}=+, \tau_{3}=-, \tau_{2}=+$ and $\sigma_{2}=-$. This innocently looking fact represents a big restriction for the possible outcomes. The two pairs with settings $(a ; b)$ and $[a ; c]$ must now have the same outcome $\sigma_{1}=+$ in Tenerife and the same is true for the two pairs $[a ; c]$ and $(b ; c)$ which now must have identical outcome $\tau_{3}=-$ in La Palma in spite of the fact that they must, in principle, correspond to two different pairs of actual measurement.

We consider now the possible correlations for the outcomes of the $M$ six-tuples of Bell (corresponding to $3 M$ measurements of pairs) that lead to different ratios of equal and different outcomes of the pairs. The number of these correlations is reduced from the original $(M+1)^{3}$ to only $2(M+1)^{2}$ different possibilities of correlations as can be seen from six-tuple (7). If we also require that $\tau_{2}=\sigma_{2}$ as Wigner did, because he argued that equal settings need to have equal outcomes, then we obtain only $(M+1)^{2}$ different possibilities of correlating equal,++-- and different,+--+ outcomes for the pairs in Bell's six-tuples (2). This latter reduction is identical to the 
reduction obtained by Bell in his inequality.

As seen from the measurement times, only 3 of the pairings of (6) do correspond to originally correlated pairs. Note that $(a ; b)$ and $[b ; a]$ cannot be treated on the same footing, although they have only interchanged settings. It is also very important to note that only one pair of Wigner's (7) is actually correlated. Each of the equal settings chosen on the same island must appear with different measurement time in Bell's sixtuple (2). In contrast, any setting that appears twice on the same island must be associated with the same measurement and, therefore, be related to the same measurement time because of Wigner's procedure involving six-tuple (5). However, it is physically impossible to actually measure two different pairs which exhibit the same setting and the same measurement time at one given location.

The ordering of the mathematical abstractions that form the subsets that Wigner chose are simply incompatible with the ordering of the original measurements in space and time. The measurement time is the only guarantee for the correct pairing of the original measurements. These facts demonstrate that Wigner oversimplified complex topological-combinatorial factors that are vital for the outcomes of his considerations. Another important fact, for the incorrectness of Wigner's pairings that arise from his 9 possibilities (6), is that the pairs with equal settings are not derived from originally correlated pairs and therefore may randomly assume all of the value-pairs,++-- , +- and -+ . They contribute to the equal outcome,++-- count, that is so important for Wigner's reasoning, only half of what Wigner actually assumed.

Thus, Wigner reduced the number of possible correlations of EPRB measurements by assuming without justification the existence of certain joint probabilities. He did not realize this fact and was, therefore, faced with the problem to explain the contradictions with actual experiments if they occurred (and as indeed were found [10] [11] [14] [15] [16]. Because Wigner was convinced that he used only set theory and Einstein's local realism, he mentioned that a violation of Einstein's separation principle would lead to “ 4 9 domains $\cdots$ of the nine measurements" corresponding to Equation (6). He thus introduced violations of Einstein's realism to explain possible future disagreements of his theory with experiments and measurements, so to speak as a deus ex machina, that would resolve contradictions; not as essential part of his proof.

We note in passing that all of our (and Wigner's) reasoning that is related to the triple of settings $a, b, c$ and Bell's inequality can be repeated with the same findings for quadruples $a, b, c, d$ and the CHSH inequality.

\section{Conclusion}

These facts show that Wigner's procedure to derive Bell's inequality is set-theoretically neither general nor sound. Wigner's work, taken in conjunction with experiments such as presented in [10] [11] [14] [15] [16] does not prove violations of Einstein's realism. It proves only that Wigner's assumptions about the existence of certain joint probabilities are incorrect. As discussed above, the essential part of Wigner's proof does not use Einstein's local realism at all. Thus, we believe to have shown beyond any reasonable doubt that Wigner derived his reduction of possible correlations and his Bell type inequality from set theoretically unjustified assumptions about the existence of joint probabilities. 


\section{References}

[1] Einstein, A., Podolsky, A. and Rosen, N. (1935) Physical Review, 47, 777-780. https://doi.org/10.1103/PhysRev.47.777

[2] Bell, J.S. (1993) Speakable and Unspeakable in Quantum Mechanics. Cambridge University Press, Cambridge.

[3] Wigner, E.P. (1970) American Journal of Physics, 38, 1005-1009. https://doi.org/10.1119/1.1976526

[4] Khrennikov, A.Yu. (2008) Entropy, 10, 19-32. https://doi.org/10.3390/entropy-e10020019

[5] Rosinger, E.E. (2014) Quantum Matter, 3, 499-504. https://doi.org/10.1166/qm.2014.1153

[6] Geurdes, J.F. (1998) Australian Journal of Physics, 51, 835-842. https://doi.org/10.1071/P97067

[7] Hess, K., De Raedt, H. and Michielsen, K. (2016) Journal of Modern Physics, 7, 1651-1660. https://doi.org/10.4236/jmp.2016.713150

[8] d'Espagnat, B. (1979) The Quantum Theory and Reality. Scientific American. https://doi.org/10.1038/scientificamerican1179-158

[9] Einstein, A. (1982) Ideas and Opinions. Three Rivers Press, New York.

[10] Aspect, A., Dalibard, J. and Roger, G. (1982) Physical Review Letters, 49, 1804-1807. https://doi.org/10.1103/PhysRevLett.49.1804

[11] Weihs, G., Jennewein, T., Simon, C., Weinfurther, H. and Zeilinger, A. (1998) Physical Review Letters, 81, 5039-5043. https://doi.org/10.1103/PhysRevLett.81.5039

[12] Fine, A. (1996) The Shaky Game: Einstein Realism and the Quantum Theory. University of Chicago Press, Chicago. https://doi.org/10.7208/chicago/9780226923260.001.0001

[13] Hess, K. (2015) Einstein Was Right! Pan Stanford Publishing, Singapore.

[14] Aguero, M.B., Hnilo, A.A., Kovalsksy, M.G. and Larotonda, M.A. (2009) European Physical Journal D, 55, 705-709. https://doi.org/10.1140/epjd/e2009-00261-y

[15] Adenier, G. (2012) AIP Conference Proceedings, 1508, 115-124. https://doi.org/10.1063/1.4773123

[16] Vistnes, A.-I. and Adenier, G. (2012) AIP Conference Proceedings, 1508, 326-333. https://doi.org/10.1063/1.4773143

[17] Zhao, S., De Raedt, H. and Michielsen, K. (2008 Foundations of Physics, 38, 322-347. https://doi.org/10.1007/s10701-008-9205-5

[18] De Raedt, H., Jin, F. and Michielsen, K. (2013) Data Analysis of Einstein-Podolsky-RosenBohm Laboratory Experiments.

[19] Leggett, A.J. and Garg, A. (1985) Physical Review Letters, 9, 857-860. https://doi.org/10.1103/PhysRevLett.54.857

[20] Feller, W. (1968) An Introduction to Probability Theory and Its Applications, Vol. 1. Wiley \& Sons New York. 
Submit or recommend next manuscript to SCIRP and we will provide best service for you:

Accepting pre-submission inquiries through Email, Facebook, LinkedIn, Twitter, etc. A wide selection of journals (inclusive of 9 subjects, more than 200 journals)

Providing 24-hour high-quality service

User-friendly online submission system

Fair and swift peer-review system

Efficient typesetting and proofreading procedure

Display of the result of downloads and visits, as well as the number of cited articles Maximum dissemination of your research work

Submit your manuscript at: http://papersubmission.scirp.org/

Or contact jmp@scirp.org 